\title{
IMPACT OF QUEUING DISCIPLINES ON THE PERFORMANCE OF MULTI-CLASS TRAFFIC IN A NETWORK
}

\author{
N.Sugirtham ${ }^{1}$, R.Sherine Jenny ${ }^{2}$ \\ ${ }^{1,2}$ Department of Electronics and Communication Engineering, Dr.Mahalingam College of Engineering and Technology, \\ Tamilnadu, India
}

\begin{abstract}
Network applications demand quality of service for enhanced call quality and increased user satisfaction. Hence, implementing queuing discipline at switches and routers in a network will govern the way packets are buffered while waiting for transmission. Queuing disciplines like first-in first-out (FIFO) queuing, priority queuing (PQ), weighted-fair queuing (WFQ), custom queuing and modified weighted round robin are more prominently deployed in network applications. These queuing disciplines help to control and manage network resources by fixing priorities for specific types of data on the network. The paper aims to analyze the quality of service parameters for various real time applications like File transfer protocol(FTP), email, database, Voice over IP(VOIP), video and web browsing,etc., and helps to optimise the use of available network resources. Moreover the quality of the network is compared using different QoS parameters such as end-to-end delay, throughput, jitter and Mean Opinion Score (MOS). This paper focuses on various queuing disciplines with low and high network traffic.
\end{abstract}

Keyword: Voice over IP, Quality of service, Jitter, Throughput, OPNET, first-in-first-out (FIFO) Queuing, Priority queuing (PQ ,Weighted-fair queuing (WFQ), custom queuing, modified weighted round robin

\section{Introduction}

LTE (Long Term Evolution) has dragged the attention of many users throughout the world. More than ever in this pandemic situation the need of digital technology has played a vital role in all pace of human kind. This circumstances has caused an intensifying demand of data usage in mobile device in terms of data transfer, streaming of media, television, video conferencing, gaming applications as well as communication through mobile video. The quality of services for IP based applications has become a significant challenge now. Wide range of research is been carried out in evaluating the performance of QoS in LAN[3,4],wireless[5], MANET[8] and Wireless sensor network[7]. As the multimedia applications are fast growing nowadays, the need to maintain a standard Quality of service(QoS) for services like surfing web, downloading pictures, and FTP is also rising.As an example voice and video services requires less delay with wide bandwidth to maintain the QoS while database, email and FTP can with stand tolerable delay. Several queuing disciplines have been designed to improve the QoS for networked multimedia applications. The issues related to multimedia applications are very well surveyed in [5].To address the issue a number of commercial tools are available [9]. OPNET is one such tool and is commercially available which has a wide-ranging library and network models. It includes GUI interface and create a real life simulation. Here we illustrate the behaviour of the network for various applications like FTP, email, database, VOIP, video and web browsing under different QoS.

The paper is structured as follows: section 2 presents an overview on various queuing discipline. Section 3 deals with design and performance evaluation. Simulation results and analysis of various QoS parameters using OPNET are discussed in Section 4. Section 5 concludes the study based on the results obtained through simulation.

\section{Queuing Discipline Overview}

This section brief on a general overview of queuing disciplines. Queuing discipline indicates the order in which the packets are serviced. QoS is the ability of any network which provides a service at a definite service level. QoS methods are based on a distribution of the available bandwidth between the various types of traffic based on the application requirements. Currently, getting a service quality agreement is easy for large and small companies. Furthermore with a a wide range of flows in the networks individuals can take advantage of this opportunity. Furthermore meeting the customers requirement on bandwidth is a major task when compared to the reservation of the bandwidth.Flows are produced by two types of applications, streaming and elastic. Audio and video applications which require real time transmission are streaming whereas elastic applications are those that require some form of digital data transfer like email, web browsing etc. Each of these flows differs in duration and volume. It is significant that it becomes difficult to provide a good level of QoS without calculating or determining the amount of resources needed to be allocated to customers. In-order to enable QoS in a network, traffic (packets) should be 
categorized into classes. Each traffic class will be treated differently based on the IP precedence for a particular traffic and DSCP (differentiated services code point) for another class. Here we have used simulation as a tool to address this issue. Modern day networks focus to provide appropriate QoS to diverse traffic classes by accommodating multiple priorities. Mechanism by which the routers prioritize and manage the traffic is said to be queuing discipline. This is achieved by algorithms said to be as queuing algorithms or scheduling algorithms.

\subsection{Scheduling Algorithms}

\subsubsection{First in-First out (FIFO)}

This is a simple technique which is widely used in internet. FIFO treats all packets equally. For a non empty queue, FIFO keeps transmitting while it tends to drop packets when the queue is full. On the other hand old packets that remained in the queue for a longer time are delivered. There are different combinations as tail drop and front drop FIFO. The authors of [6] made a study on the performance of LIFO and FIFO in depth. It is easy to implement but does not differentiate between different traffic.

\subsubsection{Priority Queuing (PQ)}

This queuing offers priority based service, wherein the lower priority queues are allowed to starve and higher priority packets are serviced here. During network congestion, in case the queue is full then low priority packets are dropped and packets with high priority are transmitted [2]. During congestion more critical data is given priority rather than the less critical data.

\subsubsection{Weighted Fair Queuing (WFQ)}

It is an extension of fair queuing and OPNET models class based weighted fair queuing. Class based weighted fair queuing is used to implement priority based service. This queuing discipline prevents the low priority queues from being ignored and they have been given chance to transmit packets [11,13]. During congestion, it allocates an output bandwidth equal to the relative weight of each traffic class.

\subsubsection{Custom Queuing(CQ)}

This queuing is preferred for applications which require minimal service. This overcomes and addresses the major weakness of priority queuing. The scheduler performs a round robin service to all the queues. A percentage of bandwidth is allocated to all the queues. When one queue is serviced and does not have any packets to sent, the scheduler moves to the next queue. Considerable amount of bandwidth is allocated to each queue $[12,13]$.

\subsubsection{Modified Weighted Round Robin (MWRR)}

It is an extension of round robin scheduling. Weights are allocated to each flow. Queues are served based on the weights. Deficit counter initialized to the queues weights is used for this purpose. Packets of variable length are serviced by MWRR[13].

Effective queue BW=(Queue weight*interface BW)/Sum of all active queue weights

\section{Performance Evaluation}

The amount of service offered by a network for an application is defined as the level of performance in terms of QoS[1] which may vary for each application. Real time applications cannot adapt to delay in traffic whereas non real time applications can tolerate delay. Interactive voice applications have a maximum delay threshold of $250 \mathrm{~ms}$ while file transfer can tolerate delay but sensitive to jitter. There are different types of traffic like FTP, VOIP, email, video, database, web browsing and mobile user considered for simulation. Hence numerous mechanisms have been proposed and deployed in existing networks to support QoS. Table 1 specifies the values assigned for various applications and load to the network in the developed simulation.

Table 1 ToS values assigned for applications

\begin{tabular}{|l|l|l|}
\hline Application & Value & $\begin{array}{l}\text { Type of service } \\
\text { assigned }\end{array}$ \\
\hline FTP & High load & Best effort \\
\hline VOIP & PCM quality & Interactive voice \\
\hline Video & $\begin{array}{l}\text { High resolution } \\
\text { video }\end{array}$ & Streaming multimedia \\
\hline Data Base & High load & Best effort \\
\hline E Mail & High load & Best effort \\
\hline Mobile & IP telephony & Interactive voice \\
\hline Web browsing & Heavy browsing & Best effort \\
\hline
\end{tabular}

Though many scheduling algorithms are proposed, we have simulated most prominent algorithms. To carryout simulation of various queuing disciplines between routers a network scenario is designed as shown in figure 1 using Riverbed modeler academic edition 17.5. This software provides modelling and simulation platform that integrates end user experience with application performance. This scenario includes routers, ethernet work station and separate servers for each traffic type. Severs are configured with buffers where packets wait in a queue. Video streaming, gaming applications, real time video and VOIP is expected to demand additional bandwidth/spectrum while FTP, email, web browsing and database use the available bandwidth. FTP is a way of uploading and downloading files to internet and a connection has to be established with the server to transfer files. VOIP deployment [10] requires provisioning for priority 
service and guarantee service. Further, it demands sufficient bandwidth guarantee for interactive operations. The behaviour of VOIP application is defined through application that includes start time, end time and repeatability. The repeatability of VOIP application is as unlimited to keep generating calls. The average message size is of VOIP is less than 100 bytes and the average jitter value should be less than $30 \mathrm{~ms}$ since voice quality is impacted by loss, latency and jitter. Whereas email application is considered as bulk and not transactional as most of these operations happen in the background and the delay incurred is not noticed by the users The average message size of this is $64 \mathrm{~KB}$ or greater. Best effort traffic includes HTTP web browsing.

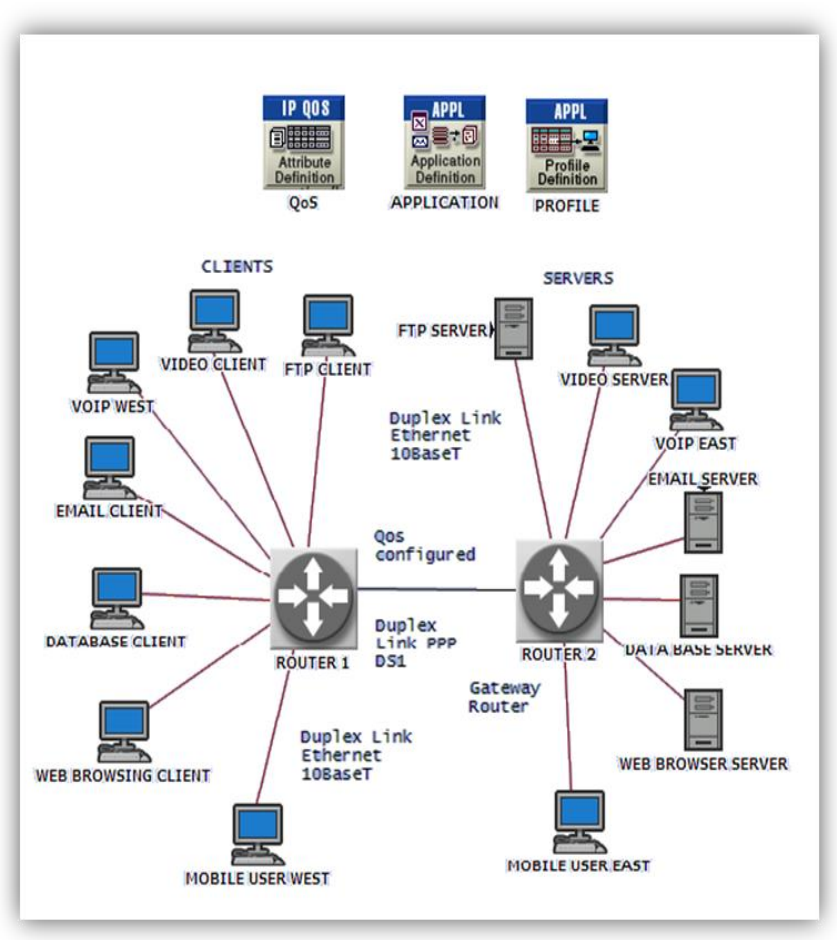

Figure 1 Scenario for queuing disciplines

All the above applications are configured in the scenario shown in figure 1 and simulated for uniform duration. Factors that influence QoS [5] include traffic dropped, average end to end delay, jitter, packet delay variation, throughput, traffic received, MOS and packet delay variation which are shown as graph. The consequences of study will lead to a solution which can ameliorate the QoS over these networks.

\subsection{Simulation Results and Performance Metrics}

Here the performance of various queuing disciplines is evaluated by taking into account the following parameters

Traffic dropped: It refers to the number of IP packets dropped when the queue gets filled up or the number of hops gets exceeds the limit.

Average end-to-end delay: This refers to the time taken or average delay incurred by the packet during transmission from source to destination. The queue condition influence this factor.
End-to-end jitter: Variation in end-to-end delay values or the variation in time between the arrival of data packets. Multimedia streaming applications performs better when the jitter is low.

Packet Delay Variation:This can be referred to as packet jitter. It is the variation in arrival time of voice packets.

Throughput: Throughput can be referred to the number of packets successfully delivered at the destination.

Traffic received: Average number of packets delivered or forwarded to all applications by the transport layer is traffic received.

Packet end-to-end Delay: This is due to the combined delay caused by the network, for performing encoding, decoding, compression, decompression, etc.

Mean Option Score (MOS) Value: This is a measure for quality of video and audio recommended by ITU-T which ranges from 1 to 5 which is shown in table 2 . And R-factor helps to access the quality of VOIP call on the network where $\mathrm{R}$-factor value is derived from latency, jitter and packet loss.

\begin{tabular}{|l|l|l|}
\hline MOS & Quality & Impairment \\
\hline 5 & Excellent & High clarity in speech \\
\hline 4 & Good & No effort needed to hear the speech \\
\hline 3 & Fair & With little effort speech is clear \\
\hline 2 & Poor & $\begin{array}{l}\text { More effort is needed to understand the } \\
\text { speech }\end{array}$ \\
\hline 1 & Bad & Communication is not possible \\
\hline
\end{tabular}

Table 2 MOS value for VOIP network as recommended by ITU-T

\section{Simulation Approach and Results}

Simulation is carried out and suitable graphs are plotted to analyze the performance of the network. From the obtained plots quantitative values can be derived that helps to measure the effect of network parameters for the considered scenario. 


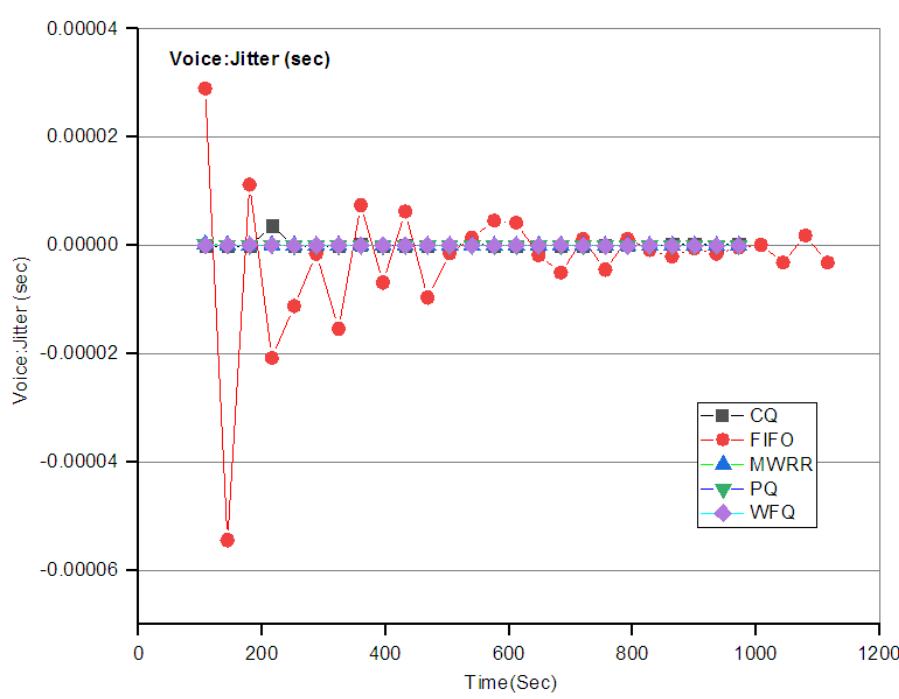

Figure 2 Jitter in seconds for voice application Jitter value is high for FIFO when compared with other disciplines which present a zero value. Therefore real time applications that cannot tolerate jitter can prefer queuing disciplines other than FIFO.

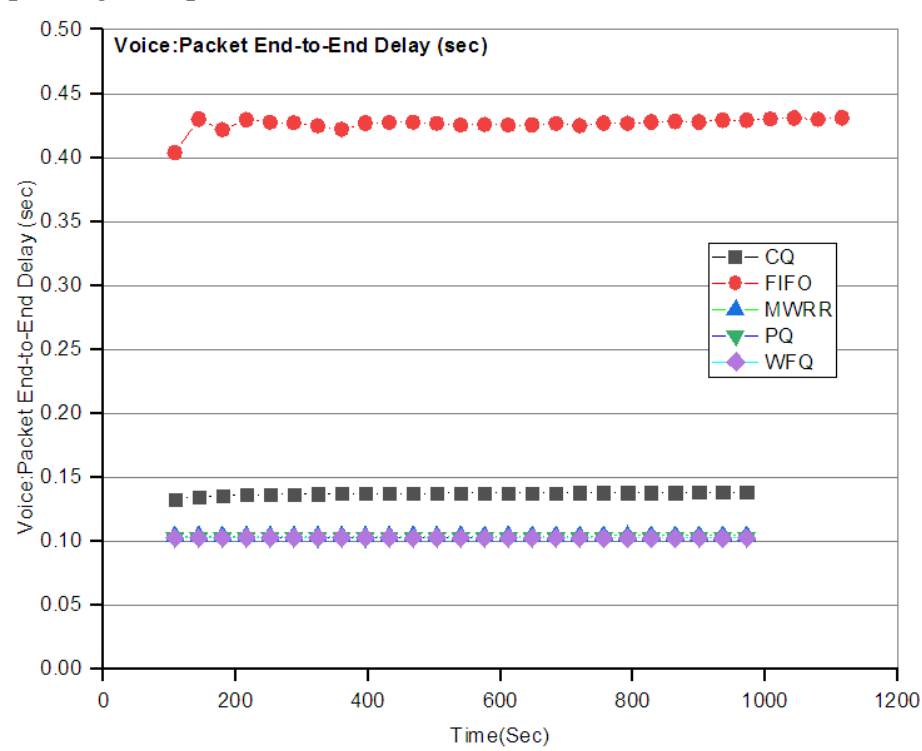

Figure 3 Packet end to end delay in seconds for voice application

From the above graph it is noticed that PQ and WFQ models are more appropriate for live video streaming with a low value of end to end delay where as FIFO holds a very high value and is not preferred.

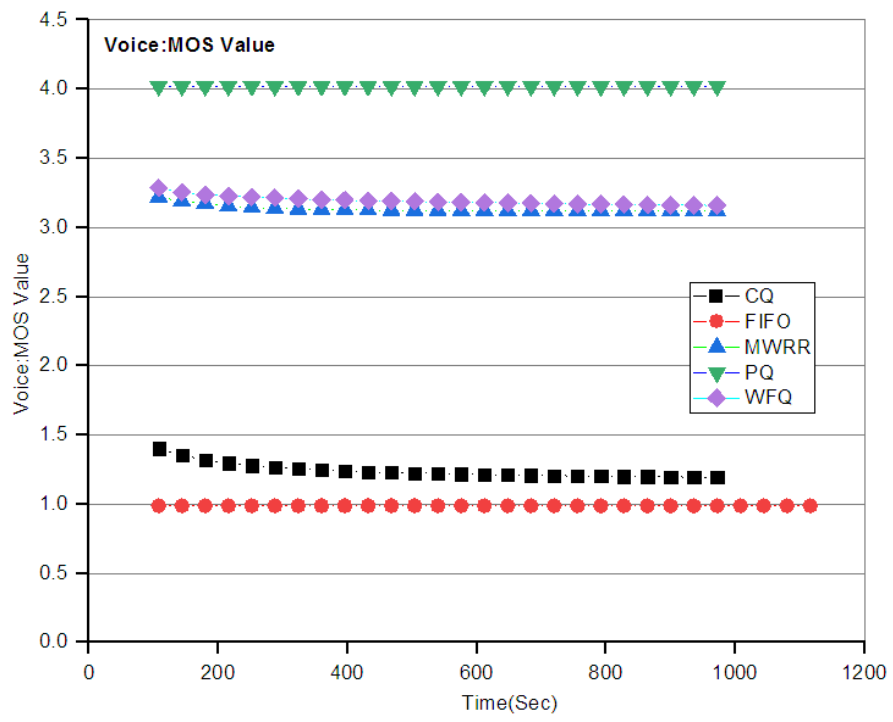

Figure 4 MOS value for VOIP network in seconds

MOS valued obtained in figure 4 can be compared with table 2 which shows that the value of PQ is 4 that is excellently well for VOIP network while the performance of FIFO is very poor.

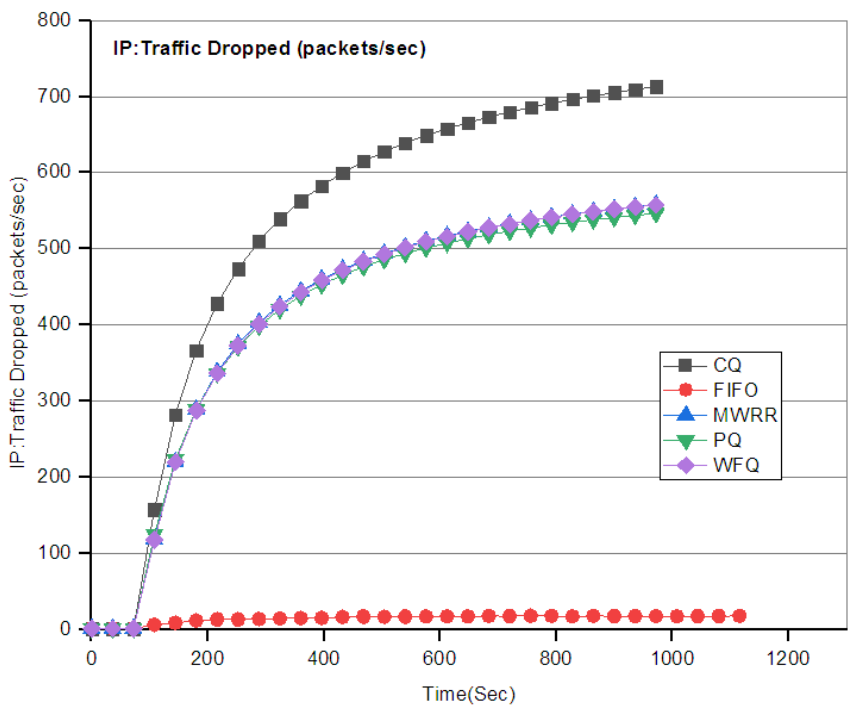

Figure 5 IP packets dropped in seconds

Traffic dropped as shown figure 5 for an IP network is high with custom queuing model whereas for FIFO queuing it holds a small value close to zero. 


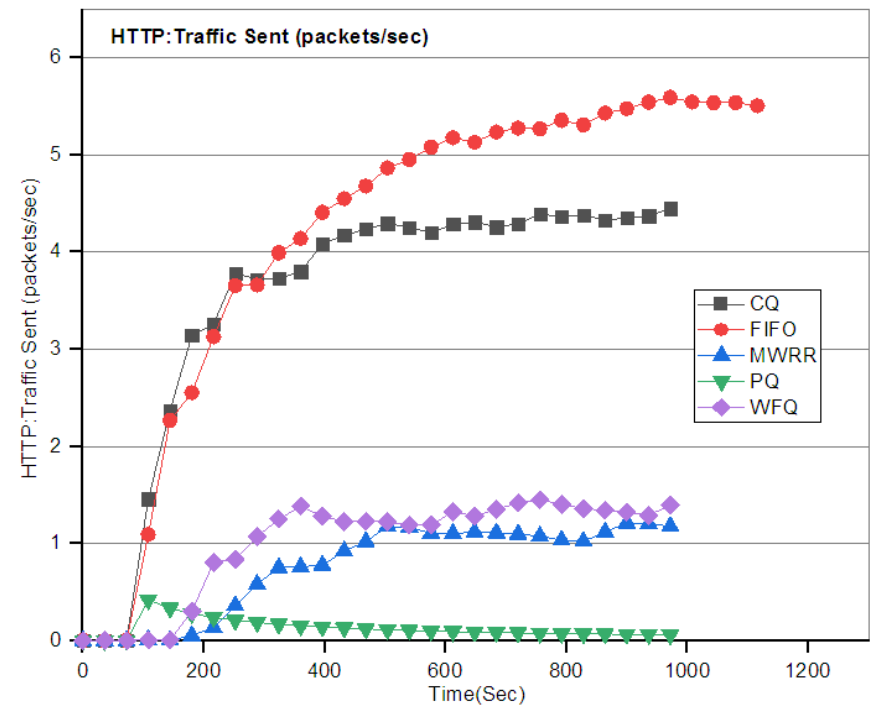

Figure 6 HTTP traffic sent in seconds

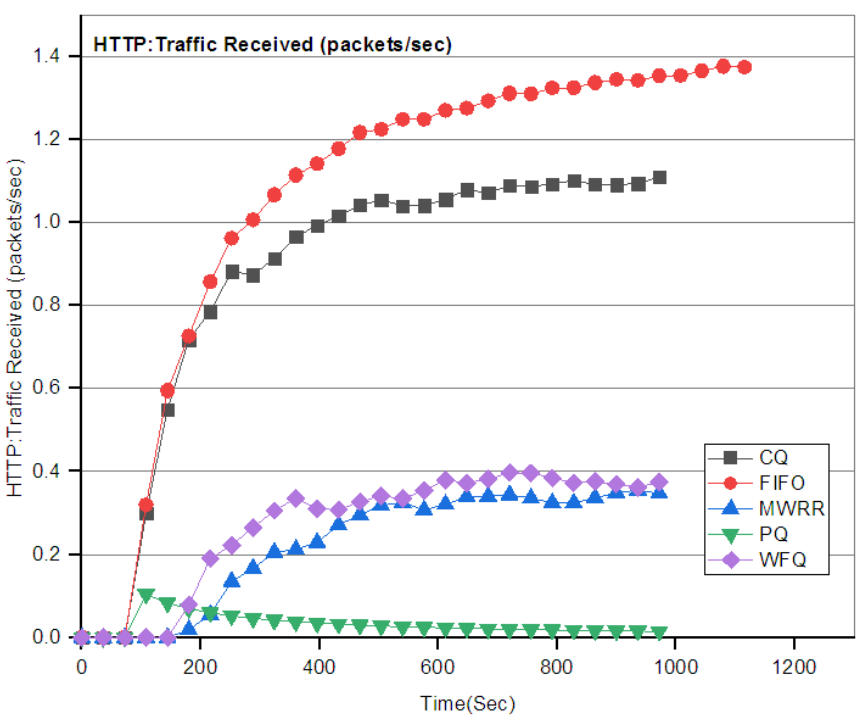

Figure 7 HTTP traffic received in seconds

Graphs obtained for HTTP traffic sent shown in figure 6 and traffic received in figure 7 depicts that PQ performs well in terms of packets received.

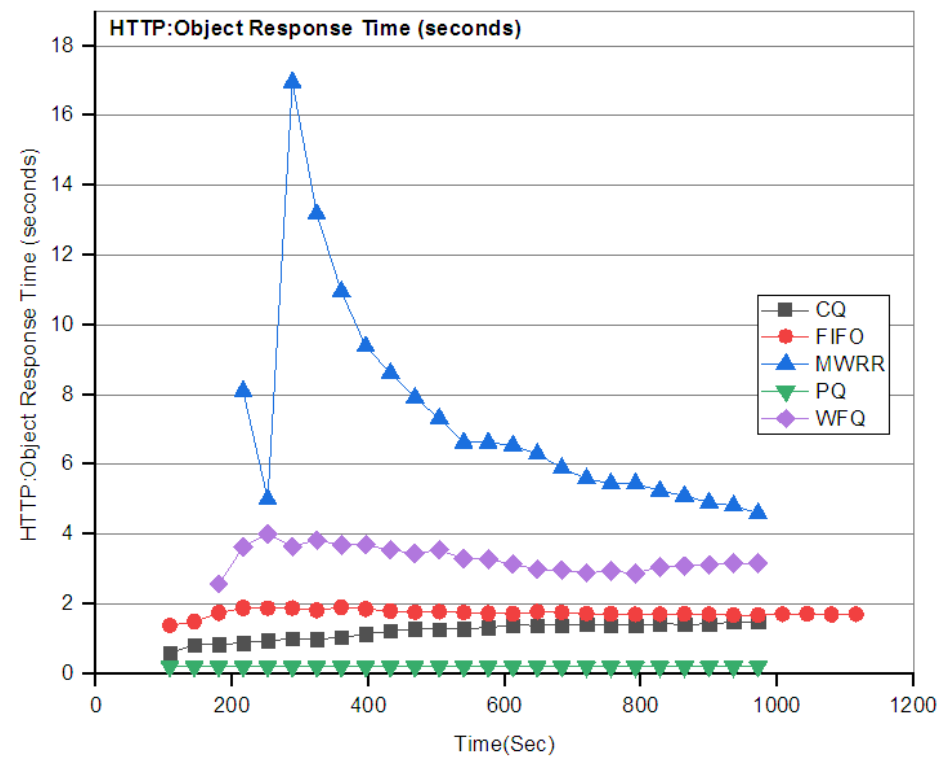

Figure 8 HTTP object response time in seconds

Performance of PQ is good for HTTP object response when compared to other disciplines as it take minimum time, a value that is very close to zero as shown in figure 8 .

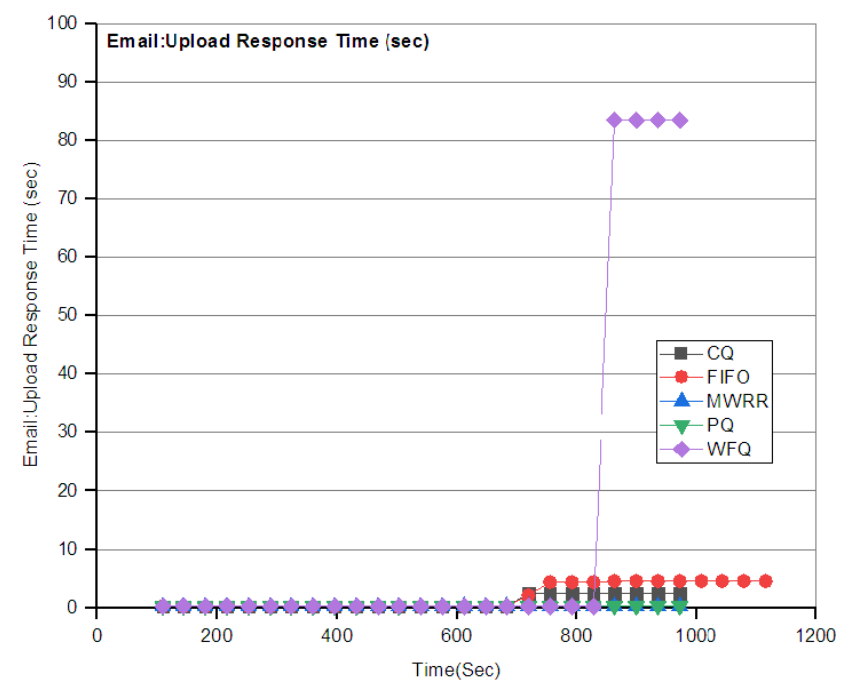

Figure 9 Upload response time for email in seconds 


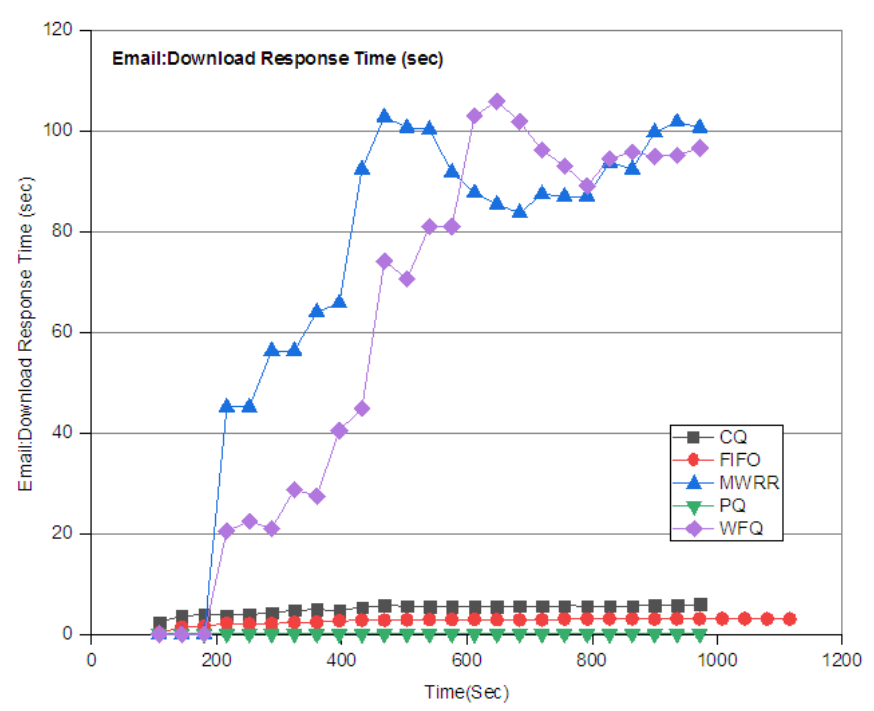

Figure 10 Download response time for email in seconds

From the graph obtained shown in figure 9 and figure 10 the upload and download response time of email application is relatively good for PQ while MWRR and WFQ show poor performance. Based on the load on the network these values slightly increase but stay within the limit. Similarly for FTP application, the upload and download response time depends on the file size. If the file size increases these times doubles.

Table 3 Point to point link parameter for queuing disciplines

\begin{tabular}{|l|l|l|l|l|l|}
\hline Parameter & FIFO & PQ & WFQ & CQ & MWRR \\
\hline $\begin{array}{l}\text { Queuing } \\
\text { delay(Sec) } \rightarrow\end{array}$ & 0.0011744 & 0.0021931 & 0.0021372 & 0.0019181 & 0.0022232 \\
\hline $\begin{array}{l}\text { Queuing } \\
\text { delay(Sec) } \leftarrow\end{array}$ & 0.0014219 & 0.0021926 & 0.0022039 & 0.0018143 & 0.0022108 \\
\hline $\begin{array}{l}\text { Throughput } \\
\text { Packets/Sec) } \rightarrow\end{array}$ & 325.55823 & 219.80654 & 211.64173 & 253.24131 & 217.55034 \\
\hline $\begin{array}{l}\text { Throughput } \\
\text { Packets/Sec) } \leftarrow\end{array}$ & 322.90656 & 219.86011 & 217.5477 & 253.42847 & 217.06631 \\
\hline Utilization $\rightarrow$ & 41.706630 & 59.249974 & 57.54014 & 59.334917 & 59.324447 \\
\hline Utilization $\leftarrow$ & 51.470152 & 59.074341 & 57.197566 & 59.146424 & 58.982882 \\
\hline
\end{tabular}

Table 3 summarizes the queuing delay, throughput and utilization of the link at the routers. The outgoing arrow $(\rightarrow)$ and incoming arrow $(\leftarrow)$ in the table represents the incoming and outgoing traffic at the link respectively. Queuing delay is experienced in the link due to packet waiting times incurred at the transmitter's queue. These measurements are considered from the entry time of a packet to the transmitter's queue to the time it takes for the last bit to be transmitted. Throughput represents the number of successful packets transmitted or received by the transmitter or receiver respectively. Utilization is the percentage usage of channel bandwidth. From the results obtained utilization of the link is high for priority queuing and custom queuing while FIFO shows minimum value. Through of FIFO queuing is low when compared to other disciplines with varying applications.

\section{Conclusion}

This paper presents a performance evaluation of queuing disciples for various applications further simulations are carried out to understand its behaviour in high traffic situations. Simulation results show that PQ and WFQ out performs other queuing disciplines in terms of jitter and packet end to end delay for VOIP application. Email upload and download response are zero for PQ when compared to others and hence PQ is best suited for email application. Extensive simulation is carried out here to assess the performance of the network. As OPNET provides practical results we are sure that the results presented here are acceptable and will help the network designers to choose an appropriate queuing discipline for a specific application. 


\section{References}

[1]. Islam, Nazrul \& Bawn, Chayan \& Hasan, Jahid \& Swapna, Asma \& Rahman, Md, (2016) "Quality of Service Analysis of Ethernet Network Based on Packet Size", Journal of Computer and Communications. 4. , 63-72.

[2]. Kohnen, C., Uberall, C., Rajarajan, M., Jäger, R. and Rakocevic, (2015) "Autonomous QoS Management and Policing in Unmanaged Local Area Networks", Journal of Computer Networks and Communications, 1-24.

[3]. Mitra, S., \& Abu-Rgheff, M, (2009) "Quality of service (QoS) issues in multimedia wireless network: A survey”. Journal of Mobile Multimedia, 5(3), 181-202.

[4]. Rukmani. P., GanesanR,(2013) "Scheduling algorithm for real time applications in mobile ad hoc network with opnet modeller", International Conference On design and manufacturing icondm, Procedia Engineering $64,94-103$.

[5]. Omar Said, (2015) "Performance evaluation of WSN management system for QoS guarantee", EURASIP Journal on Wireless Communications and Networking, vol. 2015, pp. 220.

[6]. SalahK, CalyamP, and BuhariM,(2008) "Assessing readiness of IP networks to support desktop videoconferencing using OPNET," Journal of Network and Computer Applications, vol. 31, no. 4, pp. 921943.

[7]. Nzouonta J., Ott T., Borcea C, (2009) "Impact of Queuing Discipline on Packet Delivery Latency in Ad Hoc Networks “, Elsevier Performance Evaluation Journal, Special Issue on Performance Evaluation of Wireless Ad Hoc, Sensor, and Ubiquitous Networks,.Vol. 66. No. 12.P.667-684.

[8]. Ilyashenko A., Zayats O., Muliukha V., Laboshin L.(2014) "Further Investigations of the Priority Queuing System with Preemptive Priority and Randomized Push-Out Mechanism". In: Balandin S., Andreev S., Koucheryavy Y. (eds) Internet of Things, Smart Spaces, and Next Generation Networks and Systems. NEW2AN 2014. Lecture Notes in Computer Science, vol 8638. Springer, Cham.

[9]. Vasiliadis D.C, Rizos G.E, and Vassilakis C,(2012) "Class-based weighted fair queuing scheduling on dualpriority delta networks," Journal of Computer Networks and Communications, vol. 2012, Article ID 859694, 13 pages.

[10]. Srinivas Vegesna,(2001) IP Quality of Service, Cisco press.

[11]. Configuring custom queuing [Online]. Available: http://www.cisco.com/ univercd/ cc/td/doc/ product/software/ios122/122cgcr/fqos_c/qcfcq.html
[12]. Ayoub Bahnasse, Abdelmajid Badri, Mohamed Talea, Fatima Ezzahraa Louhab, Azeddine Khiat,(2018) "Towards a New approach for automating the simulation of QoS mechanisms in a smart digital environment", Procedia Computer Science, Volume 134, Pages 227-234, ISSN 1877-0509.

[13]. Salah K, Alkhoraidly A, (2006) An opnet-based simulation approach for deploying VoIP. Int J 